\title{
Currently Used Drugs for Prevention and Treatment of Acute Organ Rejection
}

\section{Vera Maravić-Stojković* and Branislav Stojković}

Dedinje Cardiovascular Institute, Belgrade, Serbia

*Corresponding Author: Vera Maravić-Stojković, Dedinje Cardiovascular Institute, Belgrade, Serbia
Received: January 30, 2020

Published: April 29, 2020

(C) All rights are reserved by Vera Maravić-

Stojković and Branislav Stojković.

\begin{abstract}
It is clear from the knowledge gained from the ancient times that healing is not as simple process as it seems. During long periods of time, major advancements in technology, pharmacology, and medicine have enabled the extraction of countless remedial products from the plants. And not only from plants. Natural products have also been extracted from animal organs and later, synthetic equivalents with identical properties have been developed. The fascinating progress of humankind was achieved when the animal organs have become available as a drug. Nevertheless, revolutionary progress in solid organ transplantation was marked by the introduction of the cyclosporine A. Transplant immunology has become a leading science in modern medicine. Since then, solid organ transplantation has become a routine procedure accomplished with the help of protocols of immunosuppressive therapy. This article is designed as an overview of currently used immunosuppression in solid organ transplantation. Currently available groups: steroids, antimetabolites, polyclonal and monoclonal antibodies, calcineurin inhibitors, proliferation signal inhibitors, are described closely below. Each transplant center creates their own protocols. Protocols for immunosuppressive therapy offer three major strategies: induction, maintenance and therapy for treating acute rejection. The backbone of immunosuppression in organ transplantation recipient: cyclosporine A and tacrolimus, have been widely used as maintenance therapy combined with adjunctive immunosuppressant. These drugs are in use either as a prophylaxis or as the part of the maintenance immunosuppressive treatment. Induction therapy provides immunologic ablation with upstream antibody therapy as prelude to induce graft tolerance. Protocols are briefly described in the text.
\end{abstract}

Keywords: Immunosuppressive Agents; Solid Organ Transplantation

\section{Doctrinal attitude}

Since the period of the Minoan civilization (5000 B.C.), plants were used to treat infected wounds. A legend says it that the mother of the Greek hero Aeneas brought plant vitani from Mount Ida, wrapped his wounded leg with it dislodging the arrow and closing the wound [1]. Today vitani is exceptional endemic species located only on the island of Crete and it is under the European protection as a Medicinal Plant. It is clear from the knowledge gained from the ancient times that healing is not as simple process as the legend claims. During the 7 millennium-long period of time, major advancements in technology, pharmacology, and medicine have enabled the extraction of countless remedial products from the plants. And not only from plants. Natural products have also been extracted from animal organs and later, synthetic equivalents with identical properties have been developed. The fascinating progress of humankind was achieved when animal organs have become available as a drug. Blood transfusion as the first attempt of transplantation was applied two centuries ago [2]. Nevertheless, revolutionary progress in solid organ transplantation (Tx) started with the introduction of the cyclosporine A (CsA) isolated from fungus Tolypocladium inflatum. Transplant immunology has become a leading science in modern medicine.

The discovery of the immunosuppressive effect of cyclosporine A by Borel [3] in 1976 offered new hope to recipients of hepatic, cardiac, pancreatic and heart-lung transplants as acute organ rejection inevitably resulted in an early death. Surgeon Ser Roy Calne [4] was a pioneer in transplant surgery opening the new era in immunopharmacology, as well. He performed the first liver transplantation in Europe in 1968, but success was achieved only after the introduction of CsA in therapy in the early 1980s. His ingenious work continued by performing solitary heart and lung Tx or a block heart-lung transplantation. Since then, solid organ transplantation has become a routine procedure accomplished with the help of immunosuppressive therapy protocols. Increased longevity of the graft and prolonged patient's life motivated Ser Roy Calne to introduce simultaneous pancreas-kidney $\mathrm{Tx}$, and multivesicular $\mathrm{Tx}$ - the so-called cluster transplantation (stomach, intestine, pancreas, liver and kidney) in 1994. 
Every transplant center creates their own protocols with precise description of the surgical procedure, prescription of the immunosuppressive agents, the recommendation for initial doses, tapering the doses, and desired serum concentrations from surgery till the first 6 - 12 months of recovery. Protocols for immunosuppressive therapy offer three major strategies: induction immunosuppressive therapy (IISTh), maintenance immunosuppressive therapy (MISTh) and immunosuppressive therapy for treating acute rejection (ARISTh). The aim of treatment is to block activated lymphocytes, macrophages, and other immunocompetent cells of the recipient and to develop immunotolerance to the donor's antigens. A combination of 3-4 major groups of immunosuppressive drugs helps maximize the efficiency and minimize the detrimental side effects of the agents. Protocols potentiate the specificity, synergistic effect, and simultaneous actions of different immunosuppressive drugs. Solid organ transplant centers are increasingly using induction immunosuppression strategies. Induction immunosuppression involves the use of intense therapy combining four different drugs with the synergistic mechanism of action. Quadruple IISTh includes high doses steroids, antithymocyte globulin, calcineurin inhibitor (CNI), and antimetabolite introduced before or during the operation. The main objective of maintenance immunosuppression is to produce continuous host-graft tolerance. Most cardiac transplant programs use a triple therapy for MISTh consisting of corticosteroids, a CNI and an antiproliferative agent. Patients shall take the drugs during their lifetime with intention to reduce number of groups. The goal to prevent acute rejection (AR) and stimulate a tolerogenic state could be achieved within several months. Result is monotherapy, desired whenever it is possible. Corticosteroids are generally used after Tx till the end of the first year of recovery with gradual tapering of the dose. Living donor liver transplantation (LTx) recipients experience less rejection episodes comparing with deceased donor LTx and, in some cases, it is possible to exclude IS. ARISTh comprises of pulse doses of corticosteroids, and in the case of steroid-resistant AR, it could be replaced with antithymocyte globulins or Rituximab along with plasma exchange protocols (Table 1).

\section{Steroids (Ste)}

Corticosteroids have been a mainstay since early days of solid organ transplantation. It is by far most utilized non-calcineurin inhibitor immunosuppressive drugs after transplantation. Steroids produce immunosuppression by multiple mechanisms and result in a powerful and generalized anti-inflammatory response. Steroids exert their most critical immunosuppressive effect by blocking T-cell-derived and antigen-presenting cell-derived cytokine expression (IL-1, IL-3, IL-4 and IL-5, etc.). Corticosteroids continue to be used in reversing the acute rejection and in maintenance therapy. Dosing varies widely but can be summarized as follows: a bolus dose of methylprednisolone just before the surgery, 500 -

\begin{tabular}{|c|c|c|}
\hline \multicolumn{3}{|c|}{ Major groups } \\
\hline & Generic Names & Trend Names \\
\hline 1. & Corticosteroids & $\begin{array}{l}\text { Methylprednisolone, } \\
\text { Prednisolone, Pronison }\end{array}$ \\
\hline 2. & \multicolumn{2}{|c|}{ Antimetabolites } \\
\hline 2.1. & Azatioprin & Imuran \\
\hline 2.2. & Mycophenolate Mofetil & CellCept \\
\hline 2.3. & Mycophenolic Acid & Myfortic \\
\hline 3. & \multicolumn{2}{|c|}{ Polyclonal and Monoclonal Antibodies } \\
\hline 3.1. & \multicolumn{2}{|c|}{ Polyclonal Antibodies (Therapy of acute rejection) } \\
\hline 3.1.1. & Antilymphocite Globulin & ALG*, ATGAM-equine \\
\hline 3.1.2. & Antithymocyte Globulin & $\begin{array}{l}\text { ATG (Thymoglobulin) - } \\
\text { rabbit }\end{array}$ \\
\hline 3.2. & \multicolumn{2}{|c|}{ Monoclonal Antibodies (Immunoprophylaxis) } \\
\hline 3.2.1. & Muromonab-CD3 & Orthoclone-OKT3* \\
\hline 3.2.2. & Duclizumab & Zenapax* \\
\hline 3.2.3. & Basiliximab & Simulect \\
\hline 3.2.4. & Campath-1H & Alemtuzumab \\
\hline 3.2.5. & Rituximab & Mabtera \\
\hline 3.2.6. & CTLA-4-Ig & Belatacept (Nulojix) \\
\hline 4. & \multicolumn{2}{|c|}{ Calcineurin Inhibitors } \\
\hline 4.1. & Cyclosporine A & Neoral \\
\hline 4.2 & Tacrolimus & FK 506, Prograf, Advagraf \\
\hline 5. & \multicolumn{2}{|c|}{ Proliferation Signal Inhibitors } \\
\hline 5.1. & Sirolimus & Rapamune \\
\hline 5.2. & Everolimus & Certican, Afinitor \\
\hline 6. & Lymphocyte Modulation & \\
\hline 6.1. & FTY720 & Fingolimod \\
\hline
\end{tabular}

Table 1: Immunosuppressive agents currently in use [5].

Legend: ALG: Antilymphocite Globulin; ATGAM: Antilymphocite Globulin (Equine); ATG: Antithymocyte Globulin (Rabbit); CTLA4-Ig: A Fusion Protein Composed of the Fc Region of the Immunoglobulin IgG1 Fused to the Extracellular Domain of CTLA-4; OKT3: Purified Anti-Human CD3 Antibody; FK 506: Fujimycin; FTY720: Myriocin was Modified Through a Series of Steps To Yield Fingolimod, Code Named at the Time FTY720.

*Not in use.

$1000 \mathrm{mg}$, followed by rapid taper over next few weeks to minimal dose, 25 - $50 \mathrm{mg} /$ day [5,6].

\section{Antimetabolites}

The antiproliferative agents used commonly after transplantation are: azathioprine (Aza) and mycophenolate mofetil (MMF, Cell Cept). Azathioprine was the corner stone of immunosuppression therapy before cyclosporine era. Azathioprine was the first antimetabolite used in solid organ transplant but its use decreased dramatically over the time. Azathioprine is an imidazolyl deriva- 
tive of mercaptopurine and antagonizes purine metabolism. Typical dosage is $1-2 / \mathrm{mg} / \mathrm{kg} /$ day. More recently, MMF replaced Aza as the first-line antiproliferative drug, although it is several times chipper than MMF. In liver transplantation there are some limitations in MMF use, as gastrointestinal discomfort and hematological disorders limited application in initial immunosuppression (platelet count $<75 \times 10^{9} / \mathrm{L}$, diarrhea). Mycophenolate mofetil (MMF) and mycophenolic acid (MPA, Myfortic) inhibit the de novo purine nucleotide synthesis via abrogation of the inosine monophosphate dehydrogenase (IMPDH). This action leads to a blockage of DNA replication in B- and T-lymphocytes that are unable to use alternate salvage pathways. MMF and MPA are potent, selective, noncompetitive and reversible inhibitors of enzyme IMPDH. Hence, inhibits the proliferation of lymphocyte and thus prevent graft rejection, while sparing the salvage pathway $[5,6]$.

\section{Polyclonal and monoclonal antibodies}

Antibody therapy has been used as a means of delaying the introduction of maintenance therapy and/or helps facilitate the removal of an immunosuppressive agent, particularly corticosteroids. Antibody therapy can be seen as depleting or receptor modulating or both. Polyclonal Antithymocyte Globulin (ATG) are rabbit derived antibodies against several epitopes on the human T-lymphocytes (CD2, CD3, CD4, CD8, CD28 and T-cell receptor) as well against CD16 located on monocytes and natural killer cells (NK cells). These antibodies cause depletion of T-cell by apoptosis, antibody mediated cytolysis and internalization of the cell surface receptors. Basiliximab (Simulect ${ }^{\circ}$ ) is chimeric monoclonal antibody against $\alpha$-chain (CD25) IL-2 receptor T-cells. Basiliximab functions as an immunosuppressive agent specifically binding to and blocking the IL-2 receptor $\alpha$-chain on the surface on activated T-lymphocytes. Simulect is indicated for the prophylaxis of acute organ rejection in patients receiving solid organ transplantation when used as part of an immunosuppressive regimen that include CsA, MMF and corticosteroids. Belatacept received FDA-approval in June 2011 for prophylaxis of organ rejection concomitantly with Basiliximab, mycophenolate, and corticosteroids in EpsteinBarr virus seropositive kidney transplant recipients. Belatacept plus MMF or belatacept plus sirolimus provided primary immunosuppression with acceptable rates of acute rejection, improved renal function compared to a tacrolimus-based regimen, and may avoid the need for calcineurin inhibitors and corticosteroids [7]. Belatacept demonstrates a favorable cardiovascular and metabolic adverse event profile. Common adverse effects with belatacept include anemia, neutropenia, diarrhea, headache and peripheral edema. In addition, belatacept can be administered every few weeks, contrary to calcineurin inhibitors, which must be administered twice a day [7]. Rituximab (Mabtera) B-cell depleting monoclonal anti-CD20 antibody is used selectively in the treatment of humoral rejection. Rituximab destroys B cells. B lymphocytes are important cells in the immune system that help the body fight infection by producing antibodies. B cells and the antibodies they produce are also involved in process of chronic rejection after organ transplantation [8].

\section{Calcineurin inhibitors}

The early 1970s saw the introduction of a new class of immunosuppressive agents, the so-called "calcineurin inhibitors". These compounds form the backbone of immunosuppression in organ transplantation recipients. Currently, two CNIs are available: cyclosporine A and tacrolimus (FK 506, Tac). Calcineurin inhibitors, cyclosporine and tacrolimus are routinely used for immunosuppression following solid organ transplantation together with an antiproliferative agent, with or without maintenance steroids. The advantage of these drugs over cytotoxic immunosuppressants is that they act specifically on the immune system, not affecting other rapidly proliferating cells. CNIs are widely used for synergistic mechanism of action in combination with other immunosuppressive drugs. Thus, with lowering the dose of CNIs we can achieve fewer side effects, but better efficacy in immunosuppression. Due to the modern immunosuppressive therapy, the $21^{\text {st }}$ century therapy is characterized by "three $S$ ": specificity, selectivity, and synergism $[5,6]$.

Proliferation signal or mammalian target-of-rapamycin inhibitors (PSI/mTOR) include two drugs currently available for clinical use, sirolimis (Rapamune) and everolimus (Certican). Because of their dual mode of action, immunosuppressive and antiproliferative, they represent attractive options for use in solid organ transplantation. Both agents have shown efficacy for reducing the incidence of acute rejection. PSI/mTOR inhibitors work synergistically with CNIs and thus permit the minimization of CNIs without compromising efficacy. This approach is beneficial for the majority of heart transplant recipients and might provide particular assistance in specific cases, such as patients with cardiac allograft vasculopathy, malignances, post-transplantation lymphoproliferative disease (PTLD), in patients intolerant to other immunosuppressive agents. Both of PSI/mTOR inhibitors are powerful immunosuppressant but infections are serious side effects during their usage. Doses varying widely since their half-life varies from 24 to 36 hours depending on the individual patient's reaction. Usually it is $1 \mathrm{mg}$ given per day in adult population, whereas Certican has pediatric formulation of drug. The use is limited in patient having severe renal impairment since the proteinuria has been seen in nine of ten patients $[5,6]$.

\section{Lymphocyte modulation}

FTY720 is a derivative of ISP-1 (myriocin), a fungal metabolite of the Chinese herb Iscaria sinclarii as well as a structural analog of sphingosine. The new immunosuppressant FTY720 (FTY, Fingolimode) is a synthetic analogue of a natural compound. It is a novel immune modulator that prolongs allograft transplant survival in 
numerous models by inhibiting lymphocyte emigration from lymphoid organs. The mechanism of action of FTY appears to be specific and distinct from that of any other drug approved or being developed for use in solid organ transplantation. FTY rapidly and transiently reduces circulating mature lymphocytes in peripheral blood, affecting T cells more than B cells; the CD3+, CD4+ and CD8+ $\mathrm{T}$ cell lines appear to decrease the most. However, recent data indicate that FTY induces the accelerated homing of lymphocytes to lymph nodes and Peyer's patches. The lymphocyte sequestration might be mediated by an enhanced migratory response to homing chemokines.

Not only must the clinical doctor consider the whole picture for each patient, but it is also important to remember that each patient is a unique combination of influences, conditions, and susceptibilities. Nothing that happens to a transplant recipient happens for a single reason, and each patient must be addressed in the context of all factors combined in order for optimal outcomes to be achieved $[5,6]$.

If we go back to the beginning of solid organ transplantation, the first heart transplant was done Vladimir Demikhov [9] placing a new heart into the chest cavity of a dog (heterotopic). Demikhov performed the first experiment with grafting the head to the body of another dog (1954) [10]. The two-headed dog lived for a few days. One cannot deny that the picture of a German shepherd with two heads caused sensation, ranging from curiosity to shock (Figure 1).

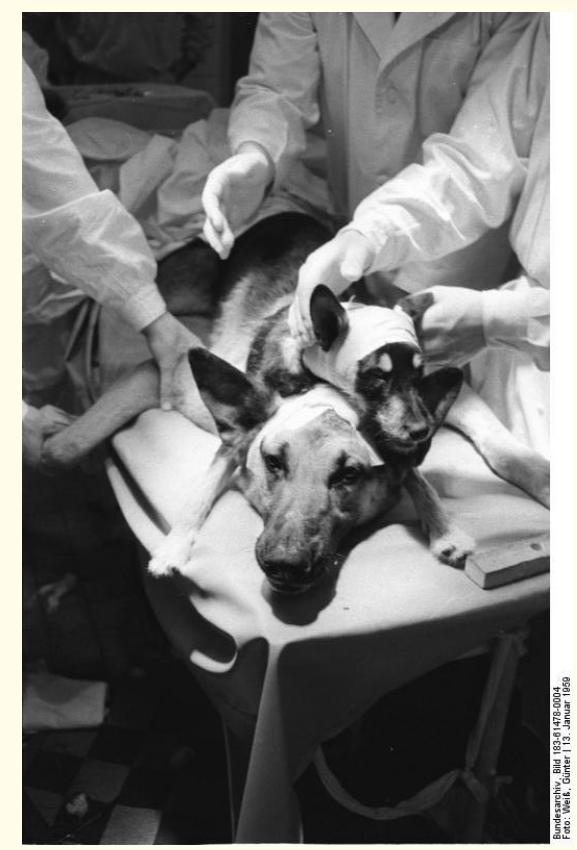

Figure 1: The last dog head transplant performed by Vladimir Demikhov on January 13, 1959 in East Germany [10].

\section{Conclusion}

However, nowadays, the head transplant is in the middle of scientific debates all around the world, from Russia to China. Surprisingly, this time the recipient is a human being. Italian neurosurgeon, professor Sergio Canavero [11] declared this debate settled in 2016 and one hundred medical doctors would assist in 36 hourslong operation. The only problem is - the law. If we go back to the Minoan time and the legend of a bizarre creature with a bull's head on human body, we could just imagine how impressive the human brain was and how extraordinary skills it possesses today.

\section{Bibliography}

1. Virgil Aeneid. 4.1-299: Latin Text, Study Questions, Commentary and Interpretative Essays (main text in Latin; commentary in English; Cambridge, UK: Open Book Publishers, 2012), by Virgil, ed. by Ingo Gildenhard (2012).

2. Yale E. "First Blood Transfusion: A History". JSTOR (2015).

3. Borel JF., et al. "Biological effects of cyclosporin A: a new antilymphocytic agent”. Agents Actions 6.4 (1976): 468-475.

4. Calne R Y. "Organ transplantation: From laboratory to clinic". British Medical Journal 291.6511 (1985): 1751-1754.

5. Maravić-Stojković V., et al. "Modern immunosuppressive agents after heart transplantation". Current Trends in Cardiology 1.2 (2007): 31-41.

6. Maravić-Stojković V. "The principles of modern immunosuppressive therapy after solid organ transplantation". SJAIT 3-4 (2011): 255-259.

7. Vincenti F., et al. "Beltacept Induction Prior to Cardiac Transplantation with Lower Intensity Maintenancand Long-therm outcomes in kidney transplantation". NEJM 374.4 (2016): 333343.

8. This study is currently recruiting participants. Prevention of Cardiac Allograft Vasculopathy Using Rituximab (Rituxan) Therapy in Cardiac Transplantation.

9. Matskeplishvili S. "Vladimir Petrovich Demikhov (19161998)". European Heart Journal 38.46 (2017): 3406-3410.

10. Konstantinov IE. "A Mystery of Vladimir P. Demikhov: The $50^{\text {th }}$ Anniversary of the First Intrathoracic Transplantation". The Annals Thoracic Surgery (1998): 1171-1177.

11. Gkasdaris G and Birbilis T. "First Human Head Transplantation: Surgically Challenging, Ethically Controversial and Historically Tempting - an Experimental Endeavor or a Scientific Landmark?" Maedica (Buchar) 14.1 (2019): 5-11. 


\section{Assets from publication with us}

- Prompt Acknowledgement after receiving the article

- Thorough Double blinded peer review

- Rapid Publication

- Issue of Publication Certificate

- High visibility of your Published work

Website: https://www.actascientific.com/

Submit Article: https://www.actascientific.com/submission.php

Email us: editor@actascientific.com

Contact us: +919182824667 